\title{
Supporting Multimedia Communication within An 802.11g Ad-hoc Network
}

\author{
Chung-Wei Lee \\ Department of CS \\ University of Illinois at Springfield
}

\author{
Jonathan Liu and Kun Chen \\ CISE Department
}

University of Florida

\author{
Yu-Chee Tseng and S.P. Kuo \\ College of Computer Science \\ National Chiao-Tung University, Taiwan
}

\begin{abstract}
By investigating the experimental results of the average throughput with the $802.11 \mathrm{~g}$ ad-hoc mode, we have collected the different performance characteristics among the indoor, the wall-penetration and the outdoor environment. Given the solid evidences from the baseline experiments, we have embedded the heuristic algorithms into the routing decisions. We have thus simulated a large area of 300 meters by 300 meters with hundreds of routing nodes. After investigating over 100 topology scenarios, the performance results indicate that our proposed scheme produces the higher-bandwidth paths for most of the cases. Even in the less-dense cases, our proposed schemes still can find the better paths with bandwidth about $30 \%$ higher than the conventional methods.
\end{abstract}

Keywords: ad-hoc networks, 802.11g, experimental study, throughput analysis, QOS routing

\section{Introduction}

Ad-hoc networks do not go through the conventional network infrastructures like access points/base stations or routers. Thus the routing function needs to be provided by the peer nodes (usually laptop computers with wireless network interface cards) in the middle of the paths. Since a truly wireless ad-hoc network will no doubt go across the mixed environments from indoor, walls to outdoor condition, it is important to obtain the baseline performance with different conditions. There are distinct differences between indoor and outdoor environments. Indoor environment always impose with ceiling and walls, which allow the signal to have more ways to propagate the signals. It is also true that indoor environments may also impose many objects (e.g., desks and chairs) which intend to scatter the signals.

On the other hand, outdoor environments do not impose ceiling, thus the signals tend to propagate like water ripples. But the natural obstacles as well as temperature and humidity condition may also play a role on the effective bandwidth that the system can achieve.
It is not clear how 802.11g ad-hoc mode performs under these different environments and how much the distance factor will contribute the overall performance. The environmental effects should be taken into consideration when it comes to real-time ad-hoc applications which require high-bandwidth support. Ideally, the routing algorithm should be also adaptive with respect to the different environments. We thus firstly investigate the average throughput between two laptop computers with the ad-hoc mode. It is our hope that different performance characteristics between indoor and outdoor settings can be collected. Then, these characteristics will be used by us to model a large-scale wireless ad-hoc network.

Though the existing netperf tool is quite popular in industry, we believe it is not a proper one for our experiments. Netperf only measures the peak performance of the connection, and it is designed for reliable environment (e.g., wire-line networks). Since the wireless environment does introduce much higher error rates for the data transmission, users hardly enjoy the peak performance reported by netperf tools. Instead, the average performance is perhaps the true one experienced by the common users. We thus developed the software tools that benchmark the average performance achieved by the ad-hoc communication. The benchmarking software emulates the constant streaming of audio/video data between two hosts. One host decides the sizes of messages in block of video/audio frames, and transmits the messages to the other host. Once the other host receives the messages, it will prepare the identical sizes of messages for sending back to the sending host. In addition, our benchmarking software discards the top $2.5 \%$ and bottom $2.5 \%$ of the measured results. Thus, our results represent the $95 \%$ confidence interval of the average performance.

Conventional thought usually predicts that the achieved bandwidth of the ad-hoc networks should be higher when the distance between the two laptop computers is short. 
However, the preliminary results indicate that this common myth is not always true. Via the experiments and analysis, we have observed that the ad-hoc mode had the worst performance within 5 meters in indoor environment. In outdoor environments, TCP and UDP have demonstrated different performance behaviors. TCP seemed to favor either short distance (e.g., 5 meters) or long distance (e.g., 25 meters). On the other hand, the best performance UDP has achieved with the distance of 10 meters. Apparently, the connection setup in outdoor environments should be also determined by the types of protocol stacks. Penetrating the walls stressed the ad-hoc mode to the limit. The performance results indicate that the distance between the routing nodes should be placed within 5 meters away. Otherwise the overall bandwidth for the connection will be significantly affected by the wall-penetration.

These unique observations indicate that the construction of an ad-hoc wireless network needs to place the distance factor in serious consideration. A proper distance range should multimedia communication such as video streaming, sufficient path bandwidth is critical to the end-to-end performance. Given the solid evidences from the baseline experiments, the routing decisions within the (multi-hop) ad-hoc networks need to be intelligent enough to guarantee the sufficient bandwidth. We have thus investigated the large area of 300 meters by 300 meters with hundreds of routing nodes. The preliminary results indicate that, even the node number is small and path selection is limited, our proposed schemes still can find the better paths with bandwidth about $30 \%$ higher than the conventional methods. With the higher density, the bandwidth of the selected paths can be 3.8 times higher compared to the conventional schemes. After investigating over 100 randomly generated topology/scenarios, the evidences indicate that our proposed schemes produce the higher-bandwidth paths.

\section{Background and Related work}

Finding the optimal routing solution to satisfying multiple quality of service (QOS) factors within the networks is a NP-complete problem [LCL05][ZLGKMT05]. Therefore, heuristic methods are usually thought to speed-up the searching for the good-enough solutions in the large-scale networks.
The wireless ad-hoc networks make the routing problem even more complex. In addition to the conventional QOS factors, the physical distances between the routing nodes with the environment factors should be jointly considered. [GJTW05] also recognized that the conventional QOS routing schemes might not fit the wireless ad-hoc networks at all. There is little literature actually addressed the concerns that we have on the distance factor, but some of the previous studies can be considered loosely related. [KH04] analyzed the detailed operations of 802.11 and proposed models to reduce the idle-time while under the collision. The work seemed to apply to the infrastructure mode instead of ad-hoc mode. Distance factor in addition to wall effect was not considered. [SWL04] focused on the load-balancing methods to reduce the control message overheads. The distributed- and hop-based AODV (Ad-hoc On-demand Distance Vector) algorithm [PRD02] was assumed in their study. Just recently, [SPH04] and [HHL06] also proposed to use hybrid methods and probability (i.e., gossip) to reduce the control message overhead due to the flooding inherited in AODV, DSR, ZRP and TORA. Our work is focused on the data communication plane. Since the control and data planes are processed separately by routing nodes, their work can jointly considered with our work to handle the control messages efficiently.

\section{Baseline Experiments}

Laptop computers are adopted to perform the actual experiments. Both of them have Pentium IV processor (with Centrino Technology), 512M memory and 40G hard disk. We then used two identical wireless adapters to be installed within the laptop to carry out this experiment. The Linksys $802.11 \mathrm{~g}$ wireless cards use $2.4-\mathrm{GHz}$ frequency with the theoretical bandwidth up to $54 \mathrm{Mbps}$. The mode has been set in ad-hoc mode with SSID "AdhocGroup". The number of channel is set to six. The subnet mask is set as 255.255.255.0 with gateway function disabled. In addition to the identical hardware, we also adopted the same operation system (Microsoft Windows XP) on the laptops. We then built our own benchmarking software on top of the TCP/UDP/IP protocol stack embedded in Windows XP operating system. 
Indoor without obstructions - We chose the CISE building basement as our experiment location in order to minimize the interference of access point of the infrastructure-mode wireless connections. Then we did the experiment at three distances: TCP, UDP with distance within $5 \mathrm{~m}$; TCP, UDP with distance at between $5 \mathrm{~m}$ and $10 \mathrm{~m}$; TCP, UDP with distance between $10-20 \mathrm{~m}$.

Outdoor without obstructions - We chose a large parking lot, and did the experiments when there are few cars parked far away in order to minimize the interference. And we also did the experiment at $5 \mathrm{~m}, 10 \mathrm{~m}, 15 \mathrm{~m} 20 \mathrm{~m}$ and $25 \mathrm{~m}$.

Penetrating wall - For this experiment, we fixed the indoor laptop's position, moved the outdoor laptop so that the distance between them is changing with distance: $5 \mathrm{~m}, 10 \mathrm{~m}$, $15 \mathrm{~m}$, and $20 \mathrm{~m}$. In between them there is a wall as the obstruction, the wireless signal has to penetrate the wall.

\section{Experimental Results and Analysis}

Indoor without Obstructions - Fig 1 depicts the results of TCP experiment performed in the indoor environment. The achieved throughput performance is indeed affected by the size of messages communicated with the two hosts. With the increasing message sizes, the throughput has been improved accordingly. For instances, with the message size of 32-Kbyte (i.e., $2^{\wedge} 5$-Kbytes in Fig 5), TCP only achieves in the range of $11 \mathrm{Mbps}$ (i.e., 19\% of the theoretical 54-Mbps peak bandwidth).

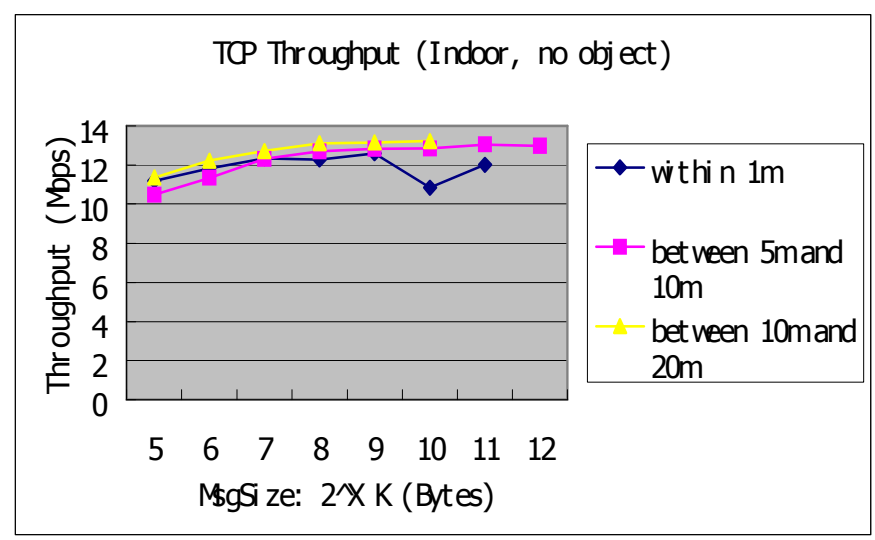

Fig.1 Indoor TCP throughput

However, with the message size of 4-Mbyte (i.e., $2^{\wedge} 12$-Kbytes in Fig 5), the throughput can be achieved to 13 Mbps. The 2-Mbps performance improvement represents the $18 \%$ increase from 32-Kbyte message size.
Just like the wire-line networks, larger messages cause smaller software overhead. Thus, the results are considered reasonable.

An interesting observation has been found related to the distance factor. It is the conventional thinking that the throughput performance should be better when the distance is shorter. The reason behind the expectation is that the time to propagate the messages to the destination is shorter. Interestingly, our measured performance results indicate the opposite trend. We expected the performance should be best with the distance less than 5 meters away. But it turns out the ad-hoc mode performs the worst compared to the distance $[5 \mathrm{~m}, 10 \mathrm{~m}]$ and $[10 \mathrm{~m}, 20 \mathrm{~m}]$. The observed performance trend demonstrates that increasing the distance does improve the achieved throughput performance.

There are perhaps a number of reasons for causing this unique performance trend. One of the reasons can be related to the multi-path propagation of the radio frequency in the physical layer. When the distance is less than 5 meters, the transmitted signal for LOS (line of sight) transmission is affected by the other reflection, diffraction and scattering radio frequencies [K85]. This unique performance trend is also demonstrated via the UDP protocol stack. The following Fig. 2 depicts the results for unit size variance between UDP and TCP. We can observe that with the increase of Unit Size, the throughput for UDP is increased correspondingly.

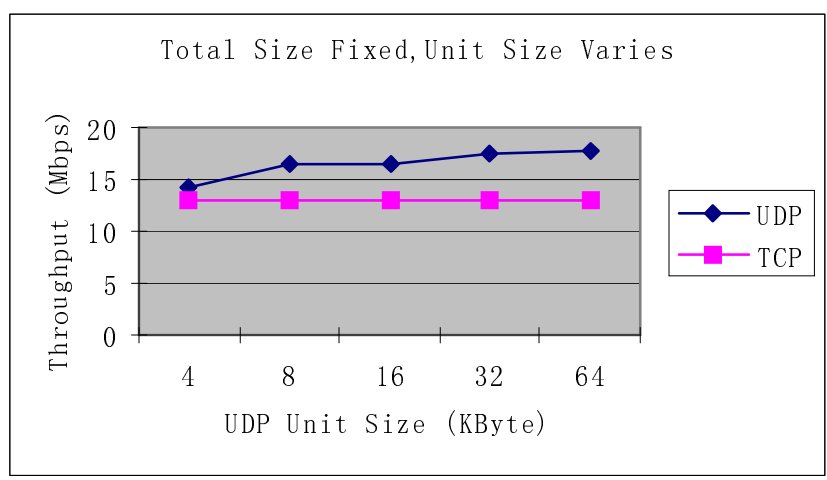

Fig.2 Unit Size influence

Without the complex congestion control as TCP does, UDP protocol stack further reduces the overhead for the different message sizes. For the same amount of data, the segmentation will be also less than the small unit size. 
The peak average throughput does reach $18 \mathrm{Mbps}$, which is an improvement of $38.5 \%$ from TCP. The UDP protocol stack almost reaches the $18.2 \mathrm{Mbps}$ when the message size is about 2 Mbyte (i.e., $2^{\wedge} 11$ KByte in Fig. 3). The similar performance trend is still observed, with the much-less bandwidth achieved when the distance is less than 1 meter. However, unlike TCP protocol stack, the achieved bandwidth seems to be quite identical when the distance is greater than 5 meters away. This unique observation makes us believe that the ad-hoc network nodes should be placed at least 5 meters away, when supporting the UDP-based applications.

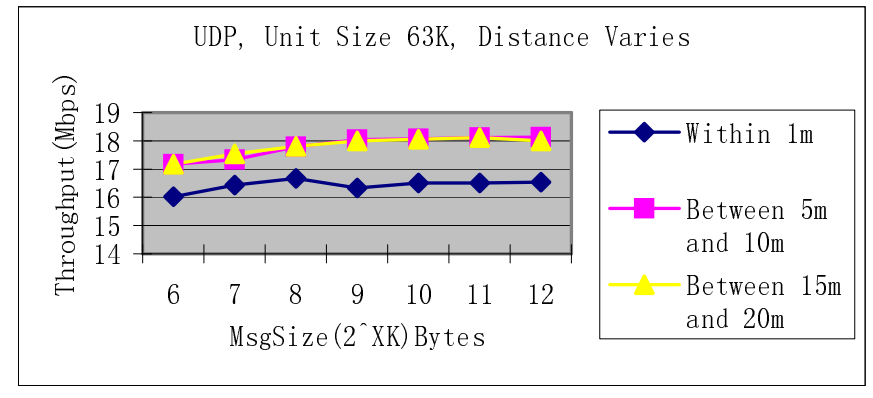

Fig. 3 Indoor UDP throughput

Outdoor without Obstructions - The TCP protocol stack's throughput performance results for outdoor environment are shown in the following Fig.4. We can observe that although the throughput results still vary due to the distance variation, the trend does not indicate the similar behavior as indoor ones with the short distance. We believe the multi-path interference has a small effect in the outdoor environments for the TCP-based applications.

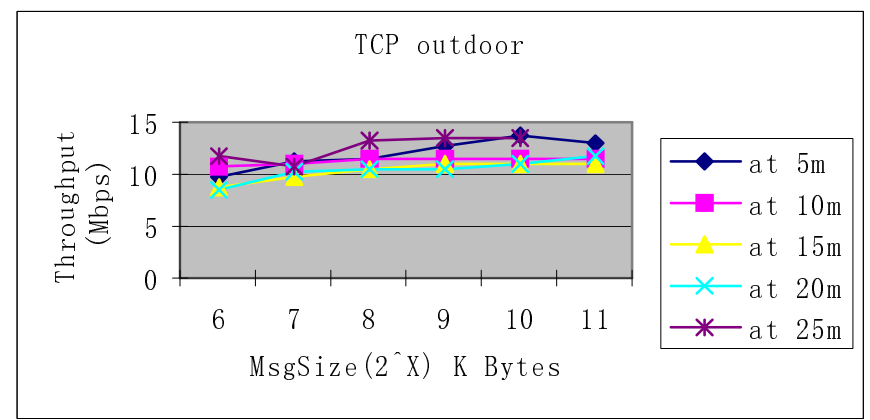

Fig. 4 Outdoor TCP Throughput

Thus, TCP protocol stack seems to perform the best when the distance is either 5 meter or 25 meters away in the outdoor environment. The TCP protocol stack seems to perform the worst when the distance is 20 meters away.
The following Fig. 5 depicts the UDP protocol stack's experimental results. The performance was further improved to reach 19.1 Mbps when the message size is large (e.g., 2 MByte). The best performance results were demonstrated with the distance was 10 meters. However, when the message sizes exceed 256 Kbytes (i.e., $2^{\wedge} 8$ Kbytes), the distance factors have limited impact on the achieved bandwidth. The overall observations thus support more placement flexibility when designing the ad-hoc networks, especially when the messages sizes are larger than 256 Kbytes. To support high-quality video streams, we also advocate for large-message sizes for the performance-guarantee among concurrent accesses.

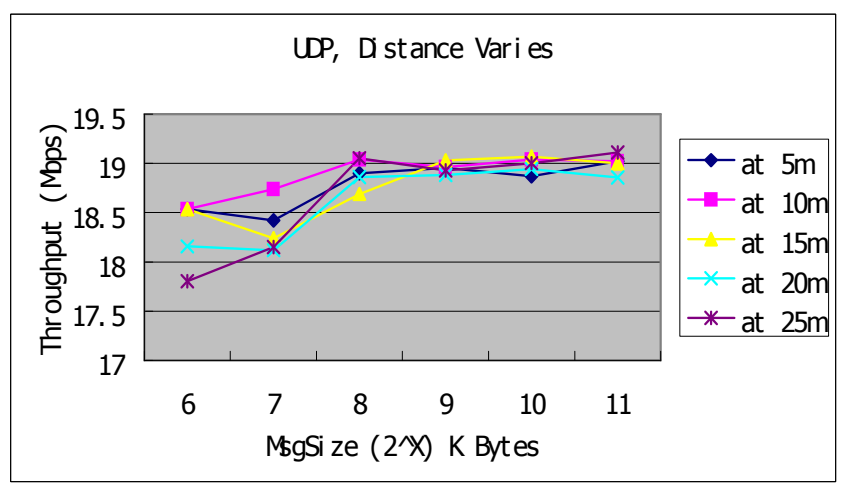

Fig.5 Output UDP Throughput

Penetrating wall- It is expected that the links between the routing nodes need to penetrate the walls between them. The penetration process needs to happen when the links are constructed to bridge the indoor and outdoor environments. After the penetration, the signals need to be strong enough for the routing nodes to detect them. Fig. 6 depicts the achieved throughput for TCP penetrating the walls.

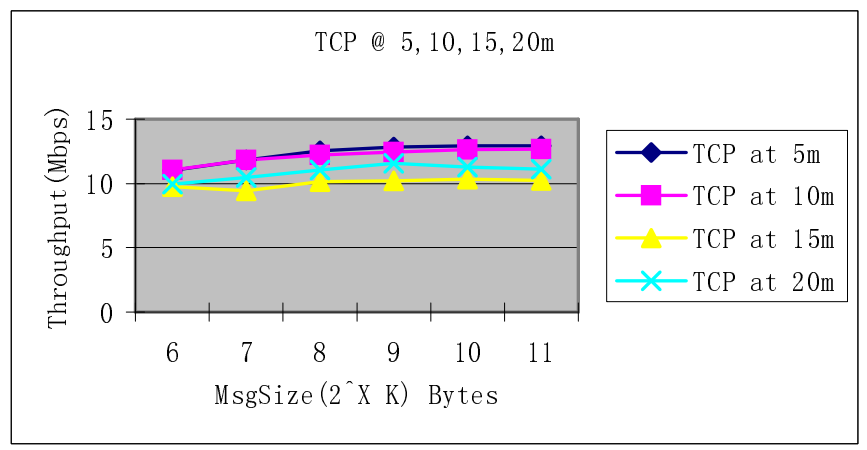

Fig. 6 TCP Penetrating Wall Results

We can observe from Fig. 6 that when the RF penetrates a wall, throughput performance is dramatically dropped. We can observe that when the distance between the nodes is increased, the achieved throughput is decreased. 
It proves that the signal strength is reduced significantly after penetrating the wall. The distance factor becomes very critical in this situation. For instance, the experimental results demonstrate that perhaps the proper distance between any two nodes should be around 5 or 10 meters. Larger-than-10 meters will cause the network bandwidth to be reduced significantly (e.g., $25 \%$ loss from 12 Mbps to 9 Mbps when the distance is 15 meters). We believe this observation is unique and important when an ad-hoc wireless network is constructed. UDP performance behavior follows the similar pattern. The worst-case performance reduction occurred with 15 meters and 512 Kbyte (i.e., $2^{\wedge} 9$ ) message size. The performance reduction is almost $44 \%$ (i.e., from $18 \mathrm{Mbps}$ to $10 \mathrm{Mbps}$ ).

\section{Large-Scale Simulations}

The discovery of IEEE 802.11g's throughput-distance relationships in its ad-hoc networking mode has significant impact in QOS routing. Since the bandwidth allocation is the top priority of the routing decision, the baseline performance results indicate that proper paths should be chosen with the consideration of the distance between any two routing nodes. However, the majority of the existing schemes seem to be lack of the joint consideration with the distance factor. For instances, many assumed the bandwidth is uniformly identical within the distance limit, thus the routing decisions favor the minimal number of hop counts. We believe, given the solid evidences from the baseline experiments, the routing decisions within the (multi-hop) ad-hoc networks should be different.

We conducted a series of QOS routing simulation experiments in order to quantitatively compare two different routing strategies. The experiments were designed to handle the large-scale cases. For instance, we believe an area of 300 meters by 300 meters with hundreds of routing nodes should be considered. Instead of comparing every existing scheme, we generalize their main concept to be the minimum-hop (MinHop) routing that searches paths with minimum number of hop count from end to end. For examples, popular mobile ad-hoc QoS routing protocols based on DSR or AODV have the similar concept since they use paths with the shortest response time.
Our proposed strategy is the maximum-throughput (MaxThroughput) routing based on our findings in $802.11 \mathrm{~g}$ 's throughput-distance relationship. It finds routes that provide the highest end-to-end path throughput (bandwidth) by jointly considering the distance factor. Since we target the multimedia communication as the long-term application goals, UDP seems to be the right choice (with application-level error correction). In the simulations, the area of 300 meters by 300 meters is specified as the boundary. Then various numbers of nodes (ranging from 10 to 200) are randomly (in terms of location) placed in this area. Having more nodes in a fixed-size area represents higher node density in a wireless ad hoc network. When all nodes are placed in the area, the link bandwidth is determined for every pair of nodes if they are within each other's transmission range. Then, the two routing strategies, MinHop and MaxThroughput, are used to obtain path (end-to-end) bandwidth $B_{i j}$ for all possible node pairs (e.g., from node $i$ to node $j$ ). Again, we believe average-case performance results better reflect the reality. Thus, for each node number/density ( $n$ ), 100 different (random) network topologies are simulated.

Our final measurement is the average path bandwidth $\left(B_{\text {avg }}\right)$ over all paths $(n(n-1) / 2)$ and 100 topologies. The following Fig. 7 shows the average path bandwidth $\left(B_{\text {avg }}\right)$ as a function of node number/density ( $n$ ) for MinHop and MaxThroughput routing. Even the node number is small (low density) and path selection is limited, MaxThroughput still can find paths with bandwidth about $30 \%$ higher than MinHop.

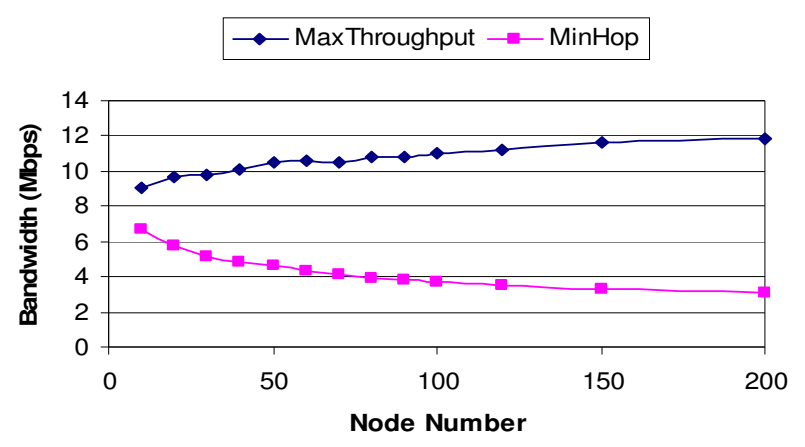

Fig. 7. Average path bandwidth as a function of node number in the network. 
As node number increases (higher density with more possible paths), MinHop tends to adopt the routing nodes that are far away from each other because this creates paths with minimum hops. However, the average bandwidth along the path is significantly reduced. On the other hand, with more selections, MaxThroughput can reach an average path bandwidth of 11.8 Mpbs when node number is 200. At the same time, MinHop has only 3.1 Mbps.

Using the simulation framework, we collect the overall results from 100 different topologies. The individual path bandwidth spans from the minimum $1 \mathrm{Mbps}$ to the maximum 18 Mbps. In addition, most importantly, the distribution of all possible path bandwidth values is shown in the following Fig. 8.

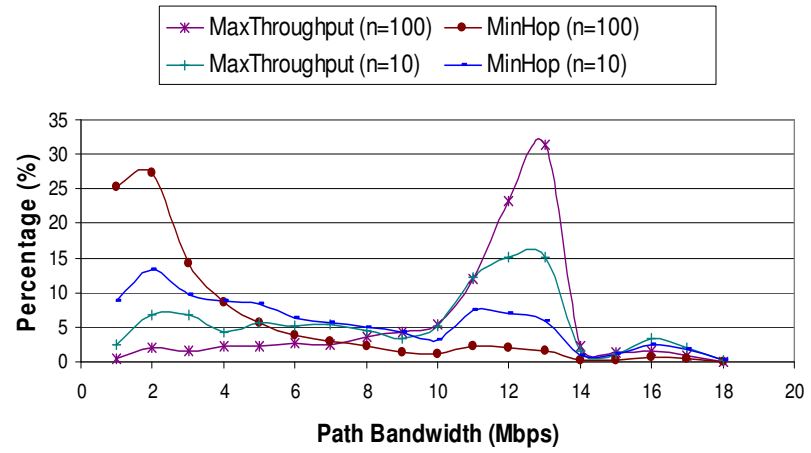

Fig. 8. Distribution of path bandwidth

Fig. 8 actually reflects the core differences from the routing heuristics. It provides the insight into the relationships among routing strategy, node density, and path bandwidth. For instances, both routing strategies show flatter distribution curves at the low density $(n=10)$ because smaller $n$ implies lacks of path choices. Nevertheless, our proposed MaxThroughput scheme still produces more paths (e.g., 17\% vs. 8\%) for higher average bandwidth (e.g., 12 Mbps). In high-density networks, the average path bandwidth is strongly affected by nature of the heuristic algorithms. The MaxThroughput scheme achieves the highest peak at $31.5 \%$ with $13 \mathrm{Mbps}$ paths. The evidences indicate that our proposed algorithm favor the higher-bandwidth paths for most of the cases. On the other hand, conventional schemes prefer short number of hops with the peak distribution of $27.3 \%$ with only $2 \mathrm{Mbps}$ paths via the MinHop routing strategy.

\section{Conclusion}

There are distinctly performance differences between indoor/outdoor environments and penetrating the walls using the ad-hoc mode. We have performed the systematic experiments to collect the performance characteristics. With the unique findings, our proposed routing schemes have improved the end-to-end bandwidth significantly. Our proposed schemes carefully choose the node-to-node routing distances (e.g., 7 meters and 20 meters in our $802.11 \mathrm{~g}$ model), therefore improves the quality of user-level applications by providing sufficient bandwidth across the selected paths.

\section{References:}

[GJTW05] R. Gupta, Z. Jia, T. Tung and J. Walrand, "Interference-aware QoS Routing (IQRouting) for Ad-Hoc Networks", Proceedings of IEEE International Conference on Global Telecommunication, 2005.

[HHL06] Z. Haas, J. Halpern and L. Li, "Gossip-Based Ad-Hoc Routing", IEEE/ACM Transactions on Networking, Vol. 14, No. 3, Jun. 2006.

[K85] H. Killen, "Modern Electronic Communication Techniques", Macmillan Publishing Company, New York, 1985.

[KH04] H. Kim and J. Hou, "Improving Protocol Capacity for UDP/TCP Traffic with Model-based Frame Scheduling in IEEE 802.11-Operated WLANs", IEEE JSAC, Vol. 22, No. 10, Dec. 2004.

[LCL05] C. Lee, R. Chow and J. Liu, "Enhancing Aggregate QoS for Video Streaming", International Journal on Computer Communications, Vol. 28, No. 18, Nov. 2005.

[PRD02] C. Perkins, E. Royer, and S. Das, "Ad-Hoc On-Demand Distance Vector (AODV) Routing", IETF Internet draft, MANET working group, Jan. 2002, draft-ietf-manet-aodv-10.txt.

[SPH04] P. Samar, M. Pearlman and Z. Haas, "Independent Zone Routing: An Adaptive Hybrid Routing Framework for Ad-Hoc Wireless Networks", IEEE/ACM Transactions on Networking, Vol. 12, No. 4, Aug. 2004.

[SWL04] J. Song, V. Wong and V. Leung, "Efficient On-Demand Routing for Mobile Ad-Hoc Wireless Access Networks", IEEE Journal of Selected Areas in Communications, Vol. 22, No. 07, Sep. 2004.

[XL04] Y. Xiao and H. Li, "Local Data Control and Admission Control for QoS Support in Wireless Ad-Hoc Networks", IEEE Transactions on Vehicular technology, Vol. 53, No. 5, Sep. 2004.

[ZLGKMT] C. Zhang, Y. Liu, W. Gong, J. Kurose, R. Moll, and D. Towsley, "On Optimal Routing with Multiple Traffic Matrices", Proceedings of IEEE International Joint Conference of Computer and Communication Societies (INFOCOM), 2005. 